\title{
Cuando sentimiento y acción confluyen en la práctica educativa. Alianzas entre educación para el desarrollo y aprendizaje servicio para una ciudadanía global
}

\author{
Yolanda Fernández Carrión, María Jesús Martínez Usarralde \\ Universitat de València, España
}

\section{Resumen}

Las problemáticas basadas en el desarrollo constituyen un tema recurrente en educación. Tras resaltar la relevancia de la Educación para el Desarrollo (EpD) como elemento fundamental en la construcción de una ciudadanía global, el objetivo de este artículo consiste en relacionarla con una cultura educativa, el Aprendizaje Servicio (ApS). Éste pretende ser, así, un análisis teórico exploratorio e interpretativo sobre las potencialidades que puede aportar el ApS en la consecución de las metas planteadas por la EpD. Esta vinculación no sólo repercute en la mejora substancial de la práctica de ambas creando sinergias positivas, sino que su universalización constituye una clara incidencia positiva en el desarrollo endógeno de los pueblos, llevando a la práctica el empoderamiento real de la ciudadanía crítica, reflexiva y global. La posibilidad de otorgar el papel relevante que posee la educación como motor de desarrollo humano está a nuestro alcance: la alianza entre EpD y APS nos muestran las claves.

\section{Palabras clave}

Aprendizaje servicio, educación para el desarrollo, empoderamiento, crítica, reflexión, solidaridad. 


\title{
When feeling and action come together in educational practice. Relations between development education and learning service for a global citizenship
}

\begin{abstract}
The difficulties based on the development are a usual topic in education. After emphasizing the relevance of the development education as an essential element for the construction of the global citizenship, the challenge stands out by its innovative intention of trying to implicate it with service learning. This one tries to be an explorative and interpretative theoretic analyses about the potentialities which can be provided by service learning in the achievement of the objectives planned by the development education. This correlation not only affects the substantial improvement of both practices by building positive synergies but also its universalization forms a clear positive influence in the endogenous development of the village people, carrying out the real empowerment of the critical, sensible and global citizenship. The possibility of giving the main role as a human developmental engine to education is within our reach and the union between development education and service learning teaches us the keys.
\end{abstract}

\section{Keywords}

Service Learning, development education, empowerment, criticism, reflexion, solidarity. 
Existe algo anterior a toda motivación de acción y es la presencia de un sentido o una consecuencia. Siempre ha existido esa expectativa impregnada en la esencia del ser humano $y$, aunque en ocasiones no se muestre de manera tan evidente, se erigen en el motor y la guía de la realidad antropocéntrica que nos condiciona. Las pulsiones que nos mueven y la razón que encauza la ilusión se cruzan en un punto muy concreto de la conciencia, se manifiesta en el espacio que se construye en un imaginario colectivo que condiciona irremediablemente la tendencia que va adquiriendo nuestro potencial. En este sentido, las diferentes teorías que difieren en su praxis sobre la fuerza de la naturaleza o del entorno en la edificación de la propia identidad convergen en la importancia que adquiere la educación como catalizadora de conocimientos que recrea una construcción mental del mundo tal y como lo concebimos, como un estado subjetivo de la realidad circundante. De esta manera, ha ido calando en la manera de entender las relaciones, de crear conocimiento científico, en el posicionamiento positivista y sesgado que la ciencia imprime en su práctica y teoría y en el paradigma en crisis del modelo de desarrollo como instrumento de progreso social. No se trata, así, de introducir temas transversales al sistema educativo formal, sino que se hace necesaria una reflexión previa a toda acción y motivación: la percepción del problema es el verdadero problema.

Uno de los temas acuciantes y quizás más controvertidos en educación es el necesario cuestionamiento eurocéntrico del modelo de desarrollo. Transformar el concepto mismo, entendiendo que desarrollo y crecimiento de manera indivisible es material y éticamente insostenible. Ésta no es más que la lógica aplastante de la evidencia, pero existe un trasfondo que sostiene todo en lo que creemos y es tan sencillo y a la vez tan complejo como la imperiosa necesidad de construirnos en equilibrio con el espacio que nos 


\section{RIDIS.}

habilita. Y cuando mencionamos a la necesidad no nos referimos tan solo al determinismo de los límites físicos y a la más que recurrente tendencia a instrumentalizar lo tangible y concreto, sino que instamos a trascender la lógica del conocimiento adquirido que se avala bajo la premisa de una falsa neutralidad, llegar más allá del uso aprendido de los elementos, valorar el carácter personal de grandes lecciones que no figuran en libros y atreverse a crear, cuestionar, sentipensar[1] (Moraes y De La Torre, 2002; Martínez Usarralde, 2011) y construir nuevos paradigmas radicalmente distintos al constructo del oasis que supone el estado de bienestar al que nos aferramos y que en el fondo justifica todo lo demás. ¿Cómo hablar de desarrollo sin saber hacia dónde? ¿Cómo abarcar la magnitud de un aprendizaje apoyado en servicios sociales sin cuestionar la finalidad del sistema en que se enmarca? Teoría y praxis en la constante espiral virtuosa del crecimiento holístico del ser.

\section{Educación para el desarrollo. El futuro de la malla curricular}

Estamos inmersos en un acelerado proceso de globalización en el que las relaciones entre los diferentes países se hacen más palpables pero cuya información aún llega parcelada, limitando la comprensión de las mismas. Esto inhibe la responsabilidad y capacidad de acción de la mayoría de la población que vive ajena a esta realidad. Es por ello necesaria la cimentación de una conciencia global que promueva el respeto de los derechos humanos en un proceso de desarrollo humano $y$ sustentable, lo que implica un cambio en las políticas y modelos de desarrollo de los países del Norte (Baselga et al., 2004).

La Educación para el Desarrollo (conocida en sus siglas EpD), recogida en el Plan Director de la Cooperación Española como estrategia sectorial, tiene como finalidad emplear el conocimiento como herramienta para cambiar la mentalidad de las personas a través 
de la comprensión de los mecanismos de interdependencia y exclusión social que existen en el mundo. Se trata de hacer uso de investigaciones, sensibilización, formación y comunicación para concienciar a la población de la necesidad de su participación en la construcción de relaciones solidarias internacionales como base para un mundo más justo (Iglesia-Caruncho, 2007).

Ese elemento evolutivo es el que se desprende de definiciones como la del Coordinadora de Organizaciones No Gubernamentales para el Desarrollo de España que asegura que la EpD debe entenderse como:

"un proceso para generar conciencias críticas, hacer a cada persona responsable y activa, con el fin de construir una sociedad civil, tanto en el Norte como en el Sur, comprometida con la solidaridad, entendida ésta como corresponsabilidad, y participativa, cuyas demandas, necesidades, preocupaciones $y$ análisis se tengan en cuenta a la hora de tomar decisiones políticas, económicas y sociales" $(2004,17)$.

Si se atiende a los cambios que ha vivido la cooperación en su escasa historia de vida, puede concluirse que la forma de entender la EpD ha sufrido, de igual modo, constantes y profundas transformaciones desde la simple sensibilización hacia situaciones ajenas, pasando por el altruismo en su defensa, cuestiones de justicia internacional, el respeto a la diversidad y el medio que compartimos y finalmente como la creación de una conciencia colectiva consciente de las interdependencias entre Norte y Sur y corresponsable de ellas. Los contenidos han ido acumulando un bagaje histórico producto de la evolución de los actores de la cooperación, principales promotores de esta educación. Actualmente comprende, bajo una concepción más crítica, la preocupación por temas actuales que suponen un desafío de igualdad: educación intercultural, educación para la paz, igualdad de género, 
educación medioambiental, educación para la ciudadanía, educación en valores, conocimiento de los condicionantes históricos de la situación presente, las relaciones de poder a nivel internacional, las causas estructurales del subdesarrollo, la realidad concreta y no tanto mediática del Sur y la capacidad crítico-reflexiva enfocada a la acción suponen una amalgama de temáticas que se concentran en el ideal del ciudadano/a cosmopolita, la ciudadanía global, sensible, ética y con iniciativa.

Según refieren Baselga et al. (2004), la EpD, como todo proceso de enseñanza-aprendizaje posee un componente cognitivo, que comprende conocimientos (economía, política, historia, ética), un componente actitudinal enfocado a la transmisión de valores como la solidaridad y la justicia y, finalmente, un componente procedimental en la que se fomenta el trabajo cooperativo, la capacidad crítica y argumentativa, y se instrumentalizan debates. Es precisamente en este último componente en el que se debiera añadir una connotación más abierta al contexto que se nutriera de la práctica en la materialización del conocimiento.

El rol del educador, para Freire, no consiste en la facilitar la aproximación a teorías que expliquen previamente la realidad circundante, sino descubrir y apropiarse de los elementos teóricos inherentes a la práctica. La construcción del conocimiento implica un movimiento dialéctico de la acción a la reflexión y de nuevo a la acción tras haber sido reflexionada, demandando la percepción particular como prisma de la realidad (Ghiso, 1996).

De esta manera, nos acercamos al Aps, principio fundamental y metodológico de una nueva manera de entender la educación que, sin duda, puede ayudarnos a la gestación de una sexta generación de EpD basada en la acción. 


\section{Aprendizaje servicio. Experiencia de una educación consciente}

El ApS se configura en estos últimos años en la educación formal como una innovación educativa que trata de combinar de manera articulada actividades de índole sociocomunitaria y el aprendizaje en las aulas como partes de una misma práctica educativa, a tenor de lo cual se han desarrollado numerosas prácticas que suscitan señalar que la teoría y la praxis se vinculan con un sentido propio más allá de la practicidad de sus acciones constituyendo axiomas inherentes al propio sistema.

Una de las definiciones más completas viene de la mano del National and Community Service Trust Act (1993) que atesora un panorama bastante amplio de la riqueza del concepto:

"El ApS es un método por el cual los estudiantes aprenden y se desarrollan a través de activa participación en un servicio cuidadosamente organizado, que se dirige y busca las necesidades de la comunidad; que coordina una escuela primaria, secundaria, la universidad o un programa de servicio de la comunidad con la comunidad; que contribuye a desarrollar la responsabilidad cívica; que está integrado en el currículum académico de los estudiantes fortaleciéndolo o se integra en los componentes educativos de los servicios comunitarios en los que están implicados los participantes; que destina tiempo previamente programado para que los estudiantes o participantes reflexionen sobre la experiencia de servicio".

Las diferencias en su definición se corresponden no sólo a los elementos que desean enfatizar sino al sentido mismo que se le concede al término. De esta manera, no existe un consenso acerca de concebir el ApS como una filosofía, una metodología o un modelo de programa social (Billig, 2000).

Podemos avanzar describiendo el ApS 


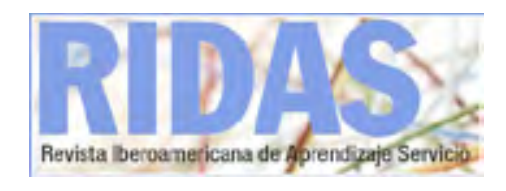

como un planteamiento educativo, basado en la articulación efectiva en la malla curricular del aprendizaje experiencial cuya calidad es retroalimentada por el servicio solidario a la comunidad, protagonizado por los estudiantes y orientado por educadores, padres y organizaciones sociales con la intención de crear conciencia crítica, participar activamente en la lucha contra las desigualdades y mejorar los recursos y habilidades de los participantes, constituyéndose el centro educativo como un innovador motor de desarrollo y empoderamiento social sensible a las necesidades del entorno.

\section{Aspectos previos para la} propuesta de acción: Aprendizaje servicio y educación para el desarrollo. El engranaje educativo del desarrollo humano

"Mientras los sistemas educativos formales propenden a dar prioridad a la adquisición de conocimientos, en detrimento de otras formas de aprendizaje, importa concebir la educación como un todo. La educación a lo largo de la vida conduce directamente a la noción de sociedad educativa, es decir, una sociedad en la que se ofrecen múltiples posibilidades de aprender, tanto en la escuela como en la vida económica, social y cultural" (Unesco (1996, 34-35).

Las actividades planteadas por el ApS, además de constituir un apoyo y refuerzo a los contenidos formales tratados en la escuela, quieren abrir un nuevo horizonte a aspectos pedagógicos tradicionalmente olvidados que incrementen la creatividad, la capacidad de imaginar, la autonomía personal y la participación activa de su propio desarrollo.

La apertura de la institución a la realidad concreta, la relación con la comunidad, el protagonismo de los estudiantes, el compromiso social vertebrado al aprendizaje y la incidencia política se completan con la intención de crear una conciencia crítica y sensible a las 
interdependencias entre los diferentes niveles geográficos y sociales aportados por la EpD. Actuando en lo local, pero contemplando lo nacional y global como parte esencial del mismo.

La verdadera fuerza transformadora que posee la unión de la EpD y ApS radica en la intención de empoderar a las personas con las que se trabaja, no solo ejerciendo incidencia sobre el contexto sino sobre las relaciones de poder que lo constituyen. De esta manera, constituyen un bastión con capacidad de erigirse como un auténtico catalizador de experiencias y reflexiones que nos conduzcan a considerarlo como el paradigma de un nuevo concepto de ciudadanía tolerante, empática, comprometida, participativa, responsable, sensible, cosmopolita, en definitiva más humana.

La EpD, históricamente, se ha basado en fundamentos teóricos de corrientes inspiradas en preocupaciones surgidas en el seno de la cooperación. De esta manera, la transmisión de este tipo de conocimientos en un ámbito puramente teórico y de forma unidireccional se diluye en la parcelación de la realidad contextual a la que es expuesto el alumnado y a la carencia, a menudo, de su retroalimentación.

Las educaciones "para" y la transversalidad de educaciones teóricas, aunque persiguen la complementariedad en el desarrollo holístico del ser, siguen labrando caminos estériles hacia utopías germinadas sobre los cimientos de un sistema obsoleto. Para ello se hace necesaria una nueva construcción de la ética bajo parámetros vivos: acercar la panorámica internacional a las aulas y devolver la experiencia práctica y educativa de procesos de desarrollo comunitarios surgidos en el seno de los centros educativos para retroalimentar esa visión globalizada. Una educación vivencial que aporta el ApS a Ia EpD, asegurando la convergencia del sentimiento y la acción en el seno de la construcción 
de un nuevo modelo de ciudadanía.

La educación formal, entre otros muchos condicionantes, está sometida a una malla curricular académica que establece tanto los contenidos teóricos, procedimentales y actitudinales a trabajar en el aula con itinerarios cerrados elaborados por personas ajenas al centro. La innovación que presupone el ApS se fundamenta en el aprendizaje basado en la acción social conjunta como fuente de transformación y compromiso. De esta manera, la introducción y acomodación del aprendizaje a través de metodologías alternativas a la establecida se convierte en una tarea compleja. La creatividad propia del ser se merma ante el patrón de enseñanzaaprendizaje de saberes predeterminados que nos inducen a sentir que la realidad es inamovible y por tanto nos inhibe del activismo social (Vallaeys, 2006). La pedagogía del ApS y de la EpD se fundamentan en la concepción de una educación para la libertad, en la que no se aceptan determinismos sino desafíos, asumiendo la incertidumbre en el proceso de aprendizaje y respetando la capacidad del alumnado a crear su propio mundo, más allá de los límites del que han heredado. Por ello, no es suficiente la incorporación de experiencias puntuales con ApS o educaciones "para", sino que es necesaria una transformación profunda del sistema educativo. Conformarse con la utilidad de ambas por separado o asumiendo su combinación como acciones inconexas es limitar el potencial transformador del aprendizaje experiencial basado en la responsabilidad social con la participación efectiva de sus ciudadanos y ciudadanas.

Un aporte, sin duda importante para el desarrollo del sentido de comunidad, es el del concepto de 'prosocialidad' que, por contraposición a las conductas antisociales, se ha instalado con fuerza en las Ciencias Sociales por su innegable capacidad de crear ambientes reductores de la violencia bajo una relación recíproca 


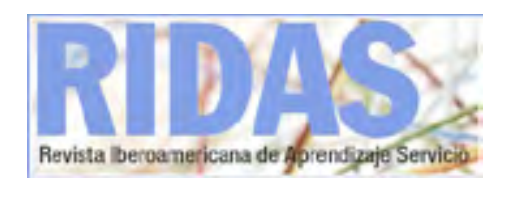

(Tapia, 2006).

Como sostiene acertadamente Cortina "es hora de pasar a la acción en la creación de una sociedad empática, solidaria, crítica y constructiva desde la apática retórica de las aulas" (1997, 37). En esa transición ejerce fuerza esta metodología que pretende transformar la manera de entender la educación, ya no para formar a futuros profesionales, sino para socializar a presentes ciudadanos, bajo valores cívicos y comprometidos con el bien comunitario.

Una de las preocupaciones en ApS, así como en EpD, es analizar la implicación que posee la ciudadanía en el desarrollo de los pueblos, entendiendo a ésta como estructuras locales interconectadas a otras redes a diferentes niveles llegando a la aldea global. Para ello es preciso entender el concepto de ciudadanía activa y participativa ligada a la libertad que le otorga el desarrollo de sus capacidades (Sen, 2000). El proceso del aprendizaje que inspira el ApS nos conduce a la necesidad de adoptar un carácter democrático en la deliberación propia de un proyecto común.

Autores como Senillosa (1998) reivindican la incidencia política como motor de desarrollo en la que el empoderamiento de la sociedad civil toma relevancia, modificando las estructuras de base que obstaculizan el desarrollo global. Como afirma Mesa (2000), este nuevo reto se afronta con dos estrategias diferenciadas: desde el Sur promoviendo el empoderamiento de los grupos más desfavorecidos y desde el Norte ejerciendo presión social y política a la idea de desarrollo excluyente, comprendiendo el origen de las dinámicas internacionales injustas para poder cambiarlas. Es interesante añadir una tercera estrategia como marco de la sexta generación de EpD donde Norte y Sur comparten la finalidad conjunta de crear una conciencia crítica y preocupada por el bienestar común. Lograr consensos equitativos a través del ejercicio activo de nuestros 


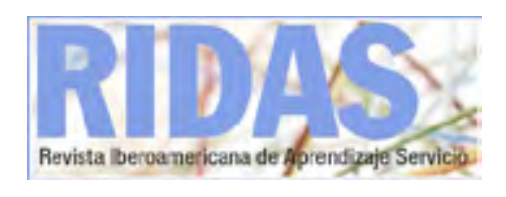

derechos como ciudadanos unidos, organizados y con iniciativa de cambio.

El ApS posee una forma de concebir la ciudadanía como elemento interdependiente y comprometida en la consecución de un bien común a través de su participación activa, informada y responsable motivada por la calidad y la calidez de una relación personal, comunicativa y cooperativa entre sus miembros, con la finalidad de lograr una convivencia armónica, asegurando el beneficio conjunto de la sociedad, lo cual actúa en consonancia con los intereses de la EpD.

La educación necesaria para ahondar en el tema de la generación de capital social no es otra que la que se promulga en los proyectos de ApS, una educación ciudadana por y para las personas participes del proceso. Este tipo de educación es entendida por Valdivieso (2003) como la gran inversión con mayúsculas para el capital social, puesto que es el vehículo para la reflexión acerca de las cuestiones fundamentales del ser humano como son la libertad, la confianza, la asociatividad, la cohesión y la democracia. Dota a los sujetos de las condiciones y capacidades necesarias para el desarrollo individual a partir de la relación con el otro.

Los dos componentes del capital social que argumentan Azqueta, Gavaldón y Margalef (2007) en su trabajo sobre educación y desarrollo, el estructural y el cognitivo, se desarrollan conjuntamente en las actividades planteadas en el ApS, pues fomenta la mejora de un clima de trabajo basado en las relaciones interpersonales y a nivel individual como una educación en valores de manera vivencial.

\section{Propuesta educativa: educación para el desarrollo de sexta generación. Claves del itinerario}

Siguiendo el modelo propuesto por el programa nacional "Escuela y Comunidad" (2001), hemos desarrollado las fases de las que 
constaría un proyecto de ApS en el marco de la EpD en un proyecto interescolar. La elección de la etapa inicial educativa responde a la necesidad de crear relaciones reales de interculturalidad y cooperación desde las primeras fases del desarrollo personal de los estudiantes en una propósito claro de aportar coherencia a la inercia de acción de la educación en valores.

En esta propuesta, la idiosincrasia del ApS se mantiene intacta y es, a su vez, absorbida por el planteamiento crítico y transformador de un modelo educativo que cuestiona el orden establecido. La capacidad transgresora del activismo aporta el elemento dinámico a las estructuras estancas sobre las que se mueve la $E p D$, en un sistema educativo cerrado e inflexible. La apuesta por el sistema formal viene amparada por la importancia de su institucionalización, lo que supondría un evidente cambio de paradigma educativo hacia concepciones más cercanas al desarrollo humano y de un marcado compromiso de transformación social.

\subsection{Preparación. Introducción a la EpD. Creación de lazos entre centros educativos del Norte y centros del Sur}

En una fase inicial, las acciones se centrarían en la creación de contactos entre ambas instituciones educativas con la finalidad de enriquecerse mutuamente a través de un intercambio horizontal de experiencias y de conocimientos autóctonos. Se trata de que se cree un vínculo en el que, a través de actividades dinámicas, se vayan conociendo los estudiantes de ambos países y abran su horizonte a la realidad del otro. En este contacto, es importante remarcar que el objetivo educativo del proceso corresponde al trabajo conjunto de análisis de los contenidos de EpD. De esta manera se crearían grupos de trabajo entre alumnos de ambos centros en los que se plantearían cuestiones críticas a trabajar de manera conjunta. Hay que tener en cuenta que las cuestiones tratadas constituirán un tema 
recurrente en los contactos que se realicen, antes, durante y tras la experiencia de ApS, pues constituye el marco teórico y estratégico de la propuesta.

Según manifiesta Tapia (2000), la intencionalidad del ApS debe ser doble: mejorar la calidad de la educación e incidir en la capacidad de acción social de las personas. Al acomodarlo al actual proyecto, tanto su sentimiento de empoderamiento como su sentido pedagógico se ve reforzado por la amplitud de perspectiva que supone la retroalimentación con otras realidades y sus experiencias, así como el bagaje adquirido por la constante evolución de la EpD, flexible y provocadora.

Como se manifiesta en el proyecto, la intención de esta propuesta es la construcción de ciudadanos del mundo, comprometidos con los desafíos que afectan a nivel internacional y en los que la acción local tiene una fuerte relevancia. La diferencia con el planteamiento actual de la EpD radica en que se trata de una educación global, no dirigida tan sólo a la sociedad de los países del Norte. En este sentido, la capacidad de análisis y el desarrollo de una conciencia crítica con la situación actual del panorama internacional se concibe como una responsabilidad de todos y una información a la que ningún tipo de educación debe quedar ajena. Dicha sensibilización, a través de un debate permanente entre los protagonistas inmersos en diferentes contextos y realidades, puede arrojar luz sobre una alternativa de desarrollo que no se puede valorar desde un solo hemisferio.

El tipo de desarrollo debe erigirse en un elemento central en las cuestiones a tratar y la capacidad de acción sobre la transformación de dicho concepto a través de la participación activa como ciudadanos que construye su propia realidad.

Qué tipo de educación para qué tipo de desarrollo constituye uno de los interrogantes básicos a los que deben enfrentarse ambos grupos y que 
fundamenta en sí la propuesta educativa. Tanto las autoridades desde su planteamiento institucional y metodológico, hasta los educadores y estudiantes desde la posición crítica de una educación en constante evolución, deben formar parte en el compromiso de un tipo de conocimiento en construcción sobre el que deben aportar con su propia experiencia.

\subsection{Planificación de la acción.} Identificación del desafío. Reconocimiento del otro.

\section{Solidaridad fuerte y realidad} glocal. Trabajo en red: centros educativos como promotores del desarrollo humano.

En esta tarea de reconocimiento es importante contemplar las particularidades de lo que se analiza, comprendiendo su cosmovisión e integrándolas como parte de su identidad, diversa y compleja y susceptible de ser compartida y escrutada.

El servicio solidario protagonizado por estudiantes pretende aprender de la práctica solidaria sobre necesidades sentidas por la propia comunidad. En este sentido, debe ajustarse la acción a los contenidos curriculares que se pretenden desarrollar pero de igual importancia es que la intervención sea entendida por los destinatarios de la misma como una forma de mejorar su calidad de vida.

Para ello, en ambos ámbitos, es necesario que los diagnósticos sean participativos. No sólo para evitar que la interpretación del contexto y sus carencias padezcan de errores en cuanto a la voluntad de sus dueños, sino también con el sentido de involucrar a los destinatarios en la acción de su propio futuro, darles voz y voto y hacerlos partícipes de una capacidad inactiva. No es que no sean capaces de lograrlo, sino que existen multitud de factores que limitan su voluntad. Darles las herramientas que permitan despertarla es una tarea fundamental de la acción social y solidaria. Para ello, debemos entender solidaridad como una relación de 
partenariado, en la que destaca la paridad en la toma de decisiones y la influencia mutua basada en la participación y el diálogo entre iguales.

Esta tendencia está siendo apoyada desde los propios organismos de desarrollo que apuestan por la capacitación de líderes de las propias comunidades como catalizadores de las iniciativas que se gestan en el propio contexto.

Partiendo de que existen varias maneras de entender la solidaridad, como sostiene Dubois (2010) en su manifiesto de la solidaridad creativa, puede distinguirse entre solidaridad débil y solidaridad fuerte. Aunque la solidaridad en sí podría definirse como un acto neutral, dependerá de las intenciones, de las evaluaciones de impacto, así como del propio proceso que sea usada para bien o para mal. A diferencia de la débil que constituye una relación de dependencia, la solidaridad fuerte promueve una relación horizontal con una obligación común bajo un compromiso real y objetivo cuya finalidad es marcada por el otro, siendo protagonista no el que se solidariza sino la situación de las personas con las que se realiza.

Esta concepción solidaria del ApS cobra fuerza en el marco de la EpD, pues apuesta por la emancipación de las personas como motor de desarrollo. En ese sentido, la aportación tanto de la conciencia crítica sobre el concepto de bienestar y el modelo de desarrollo que conlleva, así como la capacidad de acción y reivindicación como ciudadanos de derechos, desplaza a la tendencia de los gobiernos u organismos foráneos a desarrollar políticas asistencialistas.

El protagonismo de los participantes cobra especial interés en la identificación del reto que se pretende. Dicha meta no se limita a la realidad circundante, sino que supera las fronteras físicas para afrontar conjuntamente dificultades ajenas a primera vista, pero resultantes de las interdependencias interpretadas. La 
forma de entender el binomio de APS y EpD resulta clave para la evolución hacia el concepto de ciudadanía glocal[2], entendiendo sus interdependencias a nivel local pero también su implicación a nivel global en la construcción de relaciones equilibradas y justas.

Los y las estudiantes comparten vivencias, dudas, retos, experiencias, dilemas y debaten sobre la planificación de sus acciones de identificación, se valoran mutuamente y se comprenden. Redescubren su contexto y comparten sus hallazgos con sus compañeros de países diferentes porque la realidad que les devuelven también es la suya. ¿Cómo trabajar la ciudadanía global de manera aislada o parcelada? No se puede contemplar una globalización selectiva de los estereotipos como principios de una diversidad cultural, como tampoco se debe homogeneizar el ideal de progreso bajo el paradigma occidental sin pretender otras maneras de construir conocimiento. La EpD, cuyos fundamentos se nutren de derechos y obligaciones globales, debe ser universalizada. El ApS concede las claves de una apertura a la realidad social más cercana y la EpD más global.

El ApS, más allá de su dimensión pedagógica y educativa, constituye una herramienta de desarrollo local comunitario y cohesión social en la que se activan redes de tejido social bajo una atmósfera de confianza entre los participantes, plasmando una creencia generalizada de la manifiesta capacidad de generar cambios (Batlle, 2011). Tapia (2000) advierte del potencial que puede suponer un centro educativo para el desarrollo de la región como centro de investigación, creación y transferencia de conocimientos, así como agentes de socialización y como promotores no sólo del ideal de progreso si no acciones concretas de desarrollo. La tendencia habitual de la EpD a depender de las Organizaciones no Gubernamentales para el Desarrollo (ONGD) debe ser 


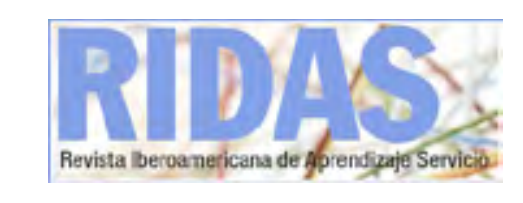

trasladada con la ayuda del ApS a los centros educativos.

Finalmente, vertebrar aprendizaje y servicio para el desarrollo resultan claves en el beneficio que puede favorecer este tipo de educación. Hay que tener en cuenta que los objetivos pedagógicos ahora contemplan las dos aportaciones en una acción conjunta. En la vinculación curricular del ApS, nos informan Puig, Batlle, Bosh y Palos (2007) que es indistinto el orden aunque es frecuente detectar una necesidad que puede ser cubierta para después decidir su aprovechamiento pedagógico, pues lograr un servicio que se adecue a los aprendizajes que pretendemos mejorar es algo más complejo. A partir de ahí, el reto consiste en identificar los contenidos académicos implícitos en el servicio que se podrían acomodar en las diferentes materias y diseñar su trabajo en el aula.

La contribución de este replanteamiento consiste en la bidireccionalidad del aprendizaje en la acomodación del servicio, pues existe un marco previo de trabajo en la capacidad de los estudiantes tanto en su conciencia crítica como de acción ante las desigualdades manifiesta en los contenidos debatidos y trabajados sobre EpD. De esta manera, el conocimiento se inserta bajo una lógica de onda, donde los círculos concéntricos van ampliando su espectro al igual que las capacidades aprendidas sobre ciudadanía global repercuten directamente en el servicio y viceversa, contribuyendo al desarrollo holístico del estudiante.

\subsection{Diseño del proyecto de aprendizaje servicio}

En esta etapa es cuando se crea el plan de acción a raíz del diagnóstico efectuado. En ella se definen las estrategias que se deben realizar para poder cumplir con las metas planteadas. Hay que tener en cuenta que los objetivos deben estar enfocados al aprendizaje y al servicio. Por un lado, definir los contenidos curriculares que se trabajan incluyendo métodos, habilidades, 
procedimientos y actitudes que se espera promover. Por otro, valorar los logros que se esperan alcanzar y definir las acciones para ello.

\subsection{Ejecución del servicio. Reflexión constante. Puesta en común}

Una vez puesto en marcha el proyecto, los actores involucrados (estudiantes, educadores, directivos, comunidad, organizaciones comunitarias, ONG, ONGD) de ambos países trabajarán en red con la finalidad de retroalimentarse y enriquecerse mutuamente de ambas experiencias. La idea es que estudiantes y educadores mantengan un contacto periódico con su semejantes para que puedan debatir y analizar sobre los desafíos que plantea la práctica social que se está llevando a cabo. La reflexión y evaluación continua del proceso se llevaría a cabo de manera conjunta, en las que se compartirían experiencias y se trataría de guiar mutuamente en la consecución de los objetivos como un verdadero trabajo de cooperación. Ambas latitudes serían corresponsables de los procesos que están llevando a cabo de forma paralela.

Un aspecto imprescindible a resaltar a lo largo del proceso consiste en prever espacios de reflexión con los estudiantes involucrados y de éstos con sus semejantes en los que se puedan manifestar las inquietudes y vivencias que están apreciando y compartir así su experiencia. Se trata de crear una plataforma de comunicación constante en la que puedan exponer sus avances en los servicios prestados, así como de los aprendizajes adquiridos y recibir la opinión de sus compañeros que están realizando la misma metodología en el mismo plazo en otra realidad distinta. De esta manera, la evaluación de la acción es individual y grupal y en ella se exploran fortalezas y debilidades y se valoran alternativas de manera conjunta para reajustar los cambios que sean necesarios.

Finalmente, en esta fase, durante el proceso son importantes los puntos 
de inflexión donde se haga un balance de lo acontecido y en los que se mantenga una comunicación constante de los avances entre los estudiantes y la comunidad y entre éstos con los educadores. No es una acción unilateral hacia sujetos con necesidades, sino una actuación conjunta a sujetos de derechos. En este sentido, la involucración de la comunidad es imprescindible, pues ha de tratarse el desarrollo local como una acción de empoderamiento comunitario, donde se contempla la participación activa de los destinatarios, alejándose de la concepción asistencialista. El diálogo entre los distintos actores, la trasparencia en la gestión, así como la rendición de cuentas se erigen en aspectos de la cooperación que se manifiestan con fuerza en esta fase. Ese diálogo es, para Freire, y según Ghiso (1996, 2), "el punto de encuentro que supera las diferencias, en el que se le puede dotar de significado a los deseos, las aspiraciones, los sueños y las esperanzas al permitir el intercambio de discursos profundamente críticos cargados de realidad, pero también de posibilidad".

\subsection{Reflexión final. Evaluación y balance del proceso y los resultados}

Evaluar en esta fase del proceso se configura como una reflexión individual y también conjunta sobre lo que ha supuesto la experiencia a todos los niveles. Es el apartado más importante, pues es cuando se sientan las bases de lo que se ha experimentado hasta el momento y se le dota de significado a lo sucedido. No sólo se deben valorar los logros e impactos de las acciones emprendidas en la comunidad o la mejora en las actitudes y habilidades de los estudiantes así como el avance en los contenidos: la evaluación implica tanto al servicio como el impacto de las acciones de manera bidireccional teniendo en cuenta a la comunidad y a los estudiantes, como a la consecución de los objetivos pedagógicos. Pero estamos hablando de aprender sobre cuestiones sociales 
y la proyección cognitivo-reflexiva debe ser ambiciosa.

Este elemento crítico, nos recuerda Tapia (2000), es el que marca la diferencia de un aprendizaje cualquiera o un servicio aislado. Su vinculación con la EpD en este proyecto refuerza la capacidad crítica de evaluar su función en la sociedad como elemento activo y transformador, promoviendo la tolerancia ante otras perspectivas vitales y la comprensión de las interdependencias sociales a nivel local, nacional y global.

Finalmente, evaluar una experiencia de este tipo en la que intervienen tantos actores, en diferentes partes del mundo, requiere prestar especial atención al proceso y contemplar aspectos cualitativos como los vínculos que se establecen entre ambos grupos. En este sentido, la sensibilidad de los educadores es de vital importancia para poder valorar el grado de esfuerzo, de implicación y compromiso de sus estudiantes y de ellos mismos en la experiencia educativa. Las expectativas deben ser realistas al tratarse de una forma experimental de educación, sin perder de vista la intención principal de crear ciudadanía global a través de un aprendizaje experiencial trasnacional donde se sientan las bases de un concepto educativo revolucionario, sin fronteras físicas ni culturales, donde la diversidad es contemplada como un factor multiplicador del alcance educativo de la cooperación. Porque el aprendizaje sirve y el servicio educa, abrirnos a una nueva forma de concebir las relaciones educativas con el desafío pedagógico puede ser el principio de un nuevo concepto de desarrollo al que nos comprometamos como ciudadanos solidarios e implicados en la construcción de un mundo más justo.

\section{Conclusión. Claves de una nueva generación de EpD A través de su alianza con el ApS}

Aunque la EpD y el ApS han
discurrido de manera independiente,
lo cierto es que poseen muchos
elementos en común y su vinculación 


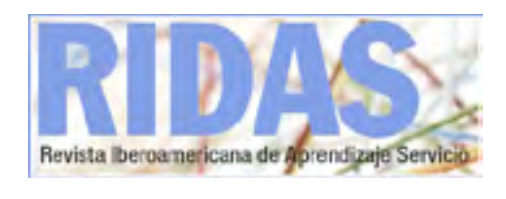

efectiva puede arrojarnos luz sobre una nueva manera de concebir la educación y la cooperación, como dos elementos interdependientes de un compromiso global de bienestar común. El ApS como una metodología generadora de buenas prácticas en consonancia con la EpD, en la que la experiencia logra emprender procesos de empoderamiento en torno a la lucha por sus derechos.

En esta tarea, es necesario un paso más en las metodologías empleadas así como en la finalidad y los fundamentos que sustentan a la EpD para dotarlo del potencial de transformación social que pretende. Las cuatro dimensiones de la EpD que expresa la Coordinadora Española de ONGD (2005) en su posicionamiento institucional (la sensibilización, la formación, la incidencia y la investigación), son posibles de manera simultánea en un nivel en el que la educación y la práctica social se combinan y se enriquecen mutuamente. Sensibilizar a través de una concienciación sobre realidades ajenas a la evidencia diaria. Emplear conocimientos que formen sobre habilidades y valores que promuevan un desarrollo humano del ser. La incidencia política en movilizaciones de acción social en las que la sociedad civil se alce ante las injusticias y en defensa de sus derechos tomando parte activa en la toma de decisiones en el planteamiento de alternativas de desarrollo a nivel local, nacional y global. $Y$ finalmente la investigación y creación de nuevos conocimientos a través de la experiencia y el análisis crítico de las situaciones analizadas y experimentadas.

Con cada vez más ímpetu toma relevancia el enfoque de promoción de una ciudadanía activa, participativa, constructiva, comprometida y preparada como base y sustento de un desarrollo humano sustentable a escala planetaria. La incidencia en el asociacionismo, la movilización, la participación, la exigencia en su discurso y acciones, así como el 


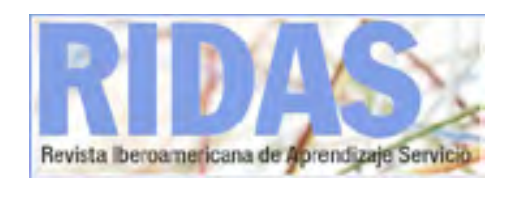

empoderamiento de las distintas capas sociales como principios en la prosperidad de un sistema que esta aún por construir, un sistema justo $y$ equitativo, incluyente y sustentable. Un sistema, en definitiva, cuyas claves se nos presentan hoy como elementos irrefutables de un nuevo orden mundial.

Baselga et al. (2004) apoyan la idea de una maduración de la EpD mediante la actuación concertada a través de redes multinivel en la que se establezca una agenda estratégica común en la que exista un reparto equilibrado de funciones. Insisten en la importancia del empoderamiento desde las organizaciones del Sur y la incidencia política desde las del Norte que altere el modelo de desarrollo dominante por una apuesta más humana, ecológica y equitativa. El sesgo radica en pretender, como manifiestan estos autores, a través de estas estrategias gestar relaciones justas entre Norte-Sur. Si bien es cierto que unos y otros poseen características diferenciadas y se sitúan en posiciones desiguales marcadas por relaciones de poder, mantener tácticas disímiles con un mismo objetivo arroja evidencias de una relación desigual de base, en la que la supremacía la sigue manteniendo una sola parte de la ecuación. El mensaje que se desprende de un discurso construido desde el Norte difícilmente puede suponer un cambio de rol en el que se pretenda dar cabida a otras opiniones. No sólo se debe cuestionar lo establecido, sino que se debe promover la construcción de alternativas desde el Sur. En este sentido, el cambio de posición de la cooperación ha sido resultado en parte por la presión del Sur en respuesta a sus evidentes fracasos, pero también a un cierto interés de recuperar su soberanía e independencia real de acuerdo a las relaciones de dependencia establecidas. Una educación para la ciudadanía global debe ser una intención por parte de todas las esferas sociales, pero también por parte de todos los países y sus 
líderes. Es importante conocer y comprender el panorama internacional sin estigmatizar por las diferencias y aceptando la relatividad del progreso en todas sus variantes. En este escenario, una sexta generación de EpD podría comprender el Aps en el Norte y en el Sur de manera coordinada, en el que se establezcan vínculos de cooperación y enriquecimiento de las diferentes experiencias. La apuesta por líderes sociales no es nada nuevo en Latinoamérica, donde se incide en el empoderamiento de las poblaciones a través del desarrollo de capacidades que le permitan desenvolverse en el sistema de cooperación internacional pero también en el propio, aprendiendo a movilizarse y reivindicar los derechos ciudadanos, ejerciendo presión al propio estado como garante de éstos.

El nuevo enfoque basado en derechos humanos que reclama la cooperación internacional sigue esta línea de dar protagonismo a los sujetos de derechos en su intención de exigir que se cumplan los acuerdos internacionales, jurídicamente vinculantes, a los sujetos de obligaciones. Pero no es una tarea única del Sur. La crisis mundial actual ha evidenciado las grietas del sistema por el que históricamente ha apostado Occidente. La participación activa de la ciudadanía y su incidencia política en la materialización de una democracia funcional y real se hace palpable en diversas dimensiones del ApS. El empoderamiento de la clase obrera, en un momento en el que se acentúa la brecha entre estamentos sociales, se torna crucial para el bienestar de la población. La alianza de la educación formal en esta tarea permite no solo la apertura a nuevas realidades y conocimientos sino la construcción de una nueva sociedad, comprometida y sensible a los retos sociales, parte concomitante de su propia realidad.

La revolución tecnológica ha contribuido a este papel de presentar a un Sur incapaz de gobernarse a sí mismo y tener éxito sin la ayuda del 
Norte, responsable en parte de su situación, que unido al atraso, la naturaleza y la ignorancia (Smillie, 1998) han condenado a la dependencia sostenida. Una mayor incidencia en crear relaciones reales entre estudiantes de diferentes partes del mundo contribuye, entendemos, a desmitificar la influencia de los medios de comunicación y a transformar los estereotipos sobre los que se construye la diversidad cultural. El ApS y su planteamiento educativo pueden contribuir significativamente a desarrollar una concepción de la EpD más allá de una estrategia de cooperación y superar los límites estancos de un sistema obsoleto en el que la influencia de las leyes del mercado toman las riendas de sus intereses. La tecnología, en este caso, se puede convertir en una aliada de la educación a través de procesos conjuntos en los que grupos de estudiantes de países del Norte y del Sur compartan sus experiencias y desafíos en la realidad de su comunidad.
Podemos concluir, subrayando que la propuesta planteada destaca por tres innovaciones simultáneas, a fin de seguir alimentando futuras investigaciones: la reinterpretación del método de ApS bajo una estrategia más amplia como es la de $E p D$, en cuya vinculación se sientan las bases de una nueva generación. Seguido a esto, la justificación de su implementación a nivel global, no sólo en países del Norte, por la singularidad de sus principios que, como comentábamos, guardan coherencia con una construcción crítica del ciudadano global de manera global. Es decir, donde ambos hemisferios tengan capacidad de decisión sobre un desarrollo a escala planetaria. Y, finalmente, por su enriquecedora posibilidad de realizar este proceso llevando a cabo una verdadera acción de cooperación, principio indiscutible de la relación que subyace al término y oportunidad única de abrirse a contemplar al otro y a uno mismo como lo que somos, ciudadanos y ciudadanas del mundo. 


\section{Referencias bibliográficas}

Azqueta, D., Gavaldón, G., Margalef, L. (2007). Educación y desarrollo: ¿capital humano o capital social?. Revista de Educación, 344, 265-283. Madrid: UNED.

Baselga, P., Ferrero, G., Boni, A., Ortega, Ma L., Mesa, M.; Nebreda, Á.; Celorio, J.J. Y Monteverde, R. (2004). La educación para el Desarrollo en el ámbito formal, espacio común de la cooperación y la educación. Propuestas para una estrategia de acción integrada. Valencia: Universidad Politécnica de Valencia.

Coordinadora de ONG de DesarrolloEspaña. Grupo de educación para el desarrollo. (2005). La educación para el desarrollo. Una estrategia de cooperación imprescindible. Madrid: CONGDE.
Cortina. A. (1997). Ciudadanos del mundo: hacia una teoría de la ciudadanía. Madrid: Alianza Editorial.

Dubois, A. (2010). Pensar un futuro para la sociedad global desde la solidaridad creativa. Revista Internacional de Estudios Vascos, Cuaderno 7, 59-95. Diputaciones Forales de Álava, Bizkaia, Gipuzkoa y Navarra.

Ghiso, A. (1996). Cinco claves éticopedagógicas de Freire. Medellín. [On line] Disponible: http://www.mon3.org/master/documentos/b_1_7_cin co_claves_freire_cip.pdf (consultado el 4/10/13).

Iglesia-Caruncho, M. (2007). Avances y retos de la cooperación española: reflexiones para una década. Madrid: Siglo XXI de España Editores.

Martínez Usarralde, M. J. (coord.) 
(2011)Sentipensar el Sur: cooperación al desarrollo y educación. Valencia: PUV.

Moraes, M., De La Torre, S. (2002). Sentipensar bajo la mirada autopoiética o cómo reencantar creativamente la educación". Creatividad y Sociedad, Revista de la Asociación para la Creatividad, 2, 4156. ASOCREA.

Puig, J. M., Batlle, R., Bosh, C., Palos, J. ( 2007$)$. Aprendizaje servicio. Educar para la ciudadanía. Barcelona: Octaedro.

Sen, A. (2000). Desarrollo y libertad. Buenos Aires: Planeta.

Senillosa, I. (1998). A new age of social movements: a fifth generation of non-governmental development organizations in the making. Development in Practice vol. 8, $\mathrm{n}{ }^{0} 1$,
55-67. London: Taylor \& Francis.

Tapia, M. (2000). La solidaridad como pedagogía. Buenos Aires: Ciudad Nueva.

Tapia, M. (2006). Aprendizaje y Servicio solidario en sistema educativo y las organizaciones juveniles. Buenos Aires: Ciudad Nueva.

Tortosa, J.M. (2001). El juego global: Maldesarrollo y pobreza en el capitalismo mundial. Barcelona: Icaria.

Valdivieso, P. (2003). Capital social, crisis de la democracia y educación ciudadana: la experiencia chilena". Revista de Sociologia e Política, 21, 13-34. Brasil: Universidad Federal de Paraná.

Vallaeys, F. (2006). El aprendizaje 
basado en proyectos sociales. $C D$ Multimedia Programa de Apoyo a Iniciativas de Responsabilidad Social, Ética y Desarrollo. Lima-Perú.

[1] Concepto empleado por diferentes autores que hace referencia a la capacidad de combinar pensamiento y emoción.

[2] Las conexiones entre lo global y lo local son sintetizadas magníficamente por Tortosa (2001) como la "glocalización" que deja ver un campo de acción desde lo local sin perder de vista lo global, pues son dos procesos interdependientes. 